\title{
DECOMPOSING THE PERSISTENCE STRUCTURE OF ISLAMIC AND GREEN CRYPTO-CURRENCIES WITH NONLINEAR STEPWISE FILTERING
}

\author{
SALIM LAHMIRI a* STELIOS BEKIROS b,c,d † \\ a ESCA School of Management, Casablanca, Morocco \\ ${ }^{\mathrm{b}}$ European University Institute, Florence, Italy \\ c Athens University of Economics and Business, Athens, Greece \\ d Rimini Centre for Economic Analysis (RCEA), Waterloo, Canada
}

\begin{abstract}
The multi-fractal chaotic dynamics of Islamic and Green crypto-currency series are investigated for the first time in econophysics literature. Specifically, we decompose and analyse the temporal signals of prices, returns, volume and volatility of Islamic and Green cryptos vis-à-vis conventional ones in a comparative manner. We introduce a multi-step resolution approach based on detrended fluctuation analysis, Generalized Hurst and Lyapunov exponents as well as fractionally integrated conditional heteroskedasticity. Moreover, various tests are employed to investigate the statistical significance of any (dis)similarities of long memory patterns, multifractality measures and chaotic dynamics observed among Islamic, Green and conventional crypto-currency markets. Our findings suggest that while the returns of Islamic and green crypto-currencies exhibit anti-persistent dynamics, their price, volatility and volume series embed high persistence compared to the conventional crypto-currencies. Further statistical testing indicates that the distributions of the chaotic parameter estimates are significantly different versus common crypto-currencies, a fact that reveals heterogeneity in multi-fractality and long memory patterns. As the Islamic and Green cryptos exhibit a distinct and more profound chaotic behaviour compared to conventional ones, their short-term predictability could further induce financial agents.
\end{abstract}

JEL Classification: 90.01 Social phenomena

Keywords: Islamic cryptocurrencies; Green cryptocurrencies; long-memory; chaos; nonlinear filtering.

\footnotetext{
${ }^{*} a$ ESCA School of Management, 7, Abou Youssef El Kindy Street, BD Moulay Youssef, Casablanca, Morocco; E-mail address: slahmiri@esca.ma

${ }^{\dagger}$ Corresponding author: ; ; ${ }^{b}$ Department of Economics, Villa La Fonte, Via delle Fontanelle, 18, I-50014, Florence, Italy; Tel.: +39 0554685925 ; Fax: +39055 4685 902; E-mail address: stelios.bekiros @eui.eu; ${ }^{c}$ Department of Acc. \& Finance, 76 Patission Str. GR10434, Athens, Greece; ${ }^{d}$ RCEA, LH3079, Wilfrid Laurier University, 75 University Ave W., ON, N2L3C5, Waterloo, Canada.
} 


\section{INTRODUCTION}

There is a vast growing interest in studying crypto-currency market dynamics. Many illustrative paradigms exist in the relevant econophysics literature. For instance, long memory was examined in [1-5], volatility was modeled in [6-9], hedging possibilities for investors were studied in [10], inherent relationships between BitCoin and AltCoin markets were documented in [11] whilst price formation was studied in [12, 13]. Furthermore, the information transmission between crypto-currency markets was documented in [14] and an analysis of high-frequency crypto-currency price series was exposed in [15]. Other works focused on long memory in volatility series [16,17], randomness in volatility [17], multi-fractal analysis [18,19], chaos and randomness [19], price forecasting via the utilization of Big data and AI techniques [20] while only recently through deep learning [21]. All previous studies [1-21] involved interesting applications of standard crypto-currency markets. However, vast awareness recently has been risen vis-à-vis Islamic [22-27] and green markets [24, 27-32] yet mostly in economics and finance literature. To the best of our knowledge, the econophysics literature on Islamic and green crypto-currency markets is significantly lacking a proper context. Therefore, to enrich the

literature on such topic, we introduce a novel stepwise decomposition approach to study long memory, fractality and chaos in prices, returns, volume and volatility series of Islamic and green crypto-currencies.

The presence of long memory may indicate the existence of a higher-order temporal dependence in the data generation process, hence potential short-term predictability. Thus, all aforementioned signals could incorporate valuable information for investors and portfolio managers, in order to optimally describe the inherent dynamics of nonlinear systems comprising the respective cryptos. It is well established that price/return, volume and volatility series always contain information regarding future profits, measures of liquidity as well as expected risk (up- and down-side). Investigating the persistence structure of Islamic and green based cryptos would help to better assess their predictability especially compared to conventional crypto-currency markets. We include an extensive comparative evaluation in this study.

Our contributions involve the introduction of a novel multi-step methodology in nonlinear filtering of first and second moments of temporal signals. We focus on long-range dependence and chaos in Islamic and green crypto-currency markets, as the latter continuously show a growing interest from digital currency investors. Interestingly, those markets have not yet been 
investigated in the econophysics literature. We extract invaluable results from the nonlinear decomposition of price, return, volume and volatility series, based on our filtering method. Moreover, our new results are compared to those obtained from a set of non-Islamic and nongreen a.k.a conventional crypto-currencies, which so far have been the center of attention for most digital traders globally.

In particular, our methodology involves in the first phase a detrended fluctuation analysis (DFA) [33] employed to measure the long memory patterns. Then, a fractionally integrated generalized autoregressive conditional heteroscedastic (FIGARCH) model [34] is used to measure any persistent characteristics embedded in the second moment series. We follow a DFA technique as it demonstrates less dependency on non-stationary and noisy data [35] and is robust to stochastic trends contaminating the signals under study. Its algorithmic convergence is also highly useful in Big data computations [36, 37]. In addition, we employ the generalized Hurst exponent [38] in order to quantify persistence at a multi-scale level. This measure allows

for the investigation of long memory at granular level thus it could prove informative on how it varies across diverse groups of crypto-currencies. The FIGARCH model, which is employed at the second and third filtering stages, is selected thanks to its flexibility and robustness vis-à-vis the distinction of short- and long-memory traits in the underlying signals. Additionally, the FIGARCH-based long-range dependence structure can be calculated under different hypotheses regarding the distribution of the filtered residuals, allowing for more degrees of freedom in estimation. Furthermore, formal statistical testing is applied on the estimated long memory parameters to check the presence of differences across Islamic, green and common cryptocurrencies. Finally, the Lyapunov exponent (LE) is used to evaluate chaos in the investigated temporal signals, in accordance with the computational methodology by Rosenstein et al [39].

The rest of the paper is organized as follows: Section 2 introduces the nonlinear multi-step dynamical filtering approach and Section 3 conducts an exhaustive empirical survey using a large pool of datasets under a Big data experiment attempt. Finally, Section 4 concludes.

\section{NONLINEAR DYNAMICAL STEPWISE FILTERING}

We utilize Detrended Fluctuations Analysis [33] to estimate long memory characteristics in the examined signals. Moreover, the Hurst exponent (denoted $H$ ) is used under a temporal context. We introduce the following approach in the first stage: 
We define the suite $x_{N}$ of the cumulative series of the signal fluctuations around the mean as

$$
x_{N}=\sum_{i=1}^{N}\left(y_{i}-\bar{y}\right)
$$

Next we divide $x_{N}$ into boxes of equal length $n$ and in each box, we fit the local trend of $x_{N}$ by a polynomial $P(n, N)$ which represents the local trend of the box. A polynomial of degree one is employed. For the given $n$ box size, we compute the root-mean-squared detrended fluctuation of the signal $x_{N}$ as

$$
F(n, N)=\sqrt{\frac{1}{N} \sum_{i=1}^{N}\left(x_{i}-P(n, N)\right)^{2}}
$$

For each of the available $n$ box size, the last step is repeated to obtain the empirical relationship between the overall fluctuation $F(n, N)$ and the box size $n$

$$
F(n, N) \propto n^{H}
$$

Then, $H$ is estimated by running a regression of $\log (F(n, N))$ on $\log (n)$. As a result, $H=0.5$ indicates that the dynamics of the original signal follow a random walk. It exhibits anti-persistent dynamics when $0<H<0.5$ whilst persistent dynamics are captured when $0.5<H<1$. Finally, when $H \geq 1$, temporal autocorrelations exist but they cease to be a power-law form [33].

The generalized Hurst exponent (GHE) [38] measures the multi-scaling properties of each crypto-currency time series, whereby long memory is computed at different scales to describe its short and long variations. Generally, for a given signal $S(t)$ defined at discrete time intervals $\mathrm{t}=v, 2 v, \ldots T$ over a period $T$ (integer multiple of $v$ ), the $q$ th-order moments of the distribution of the statistical evolution of $S(t)$ is given by [38]

$$
K_{q}(d)=\frac{\left\langle\|S(t+d)-S(t)\|^{q}\right\rangle}{\left\langle|S(t)|^{q}\right\rangle}
$$

where $d \in\left[v, d_{\max }\right]$ is a time interval and $d_{\max }$ is its predetermined upper limit. The GHE given by $H(q)$ is defined from the scaling behavior of $K_{q}(d)$ based on the following empirical relation

$$
K_{q}(d) \propto=\left(\frac{d}{v}\right)^{q H(q)}
$$

In case $K_{q}(d)$ and $d$ satisfy a linear relationship for a given order $q$ in log-log scale, the Hurst exponent $H(q)$ can be estimated by applying a linear regression of $\log \left(K_{q}(d)\right)$ versus $\log (d)$. 
Under this framework, $H(q)$ describes th long memory dependence or persistence in the original signal $S(t)$. Hence, the multi-scaling structure of the signal $S(t)$ is related to different orders $q$ of the exponent $H(q)$. In our study, the range of $q$ is fixed within the interval from -10 to $10(q \neq 0)$. The parameters $v$ and $d_{\max }$ are respectively set to 5 and 19 .

The second stage incorporates second-moment modeling. For this, the fractionally integrated generalized autoregressive conditional heteroscedastic (FIGARCH) model [34] is employed. It embeds memory traits in estimated signal volatility. For a dynamic signal $r_{t}$ the plain GARCH $(1,1)$ model, adopted for simplicity herein, is expressed as

$r_{t}=\mu+\varepsilon_{t}$

where, $\varepsilon_{t}=h_{t}^{0.5} \eta_{t} \quad(7)$ and

$h_{t}=\omega+\alpha \varepsilon_{t-1}^{2}+\beta h_{t-1}$

where $\mu$ is the conditional mean, $h$ is the conditional standard deviation and $\eta \sim \mathrm{N}(0,1)$. Subsequently, the FIGARCH $(1, d, 1)$ is given by

$$
h_{t}=\omega+\beta h_{t-1}+\left[1-(1-\beta L)^{-1}(1-\phi L)(1-L)^{d}\right] \varepsilon_{t}^{2}
$$

where $d$ is the fractional integration parameter used to describe long-range memory, with $0 \leq d$ $\leq 1, \omega>0, \phi, \beta<1$, and $L$ as the lag operator. If $0<d<1$ then intermediate ranges of persistence are allowed, while for $d=1$ the volatility series exhibit full integrated persistence. When $d=0$ the series decay with a geometric rate. We estimate the FIGARCH model parameters via conventional maximum likelihood method under the hypothesis that the error term obeys a $t$ skewed distribution in order to take into account asymmetric/leverage effects. The volatility series of price and volume are estimated as first log-differences.

In the third phase, we adopt the algorithm of Rosenstein et al [39] to estimate the Lyapunov exponent (LE). The main advantages of our approach include accuracy, robustness to small and noisy data sets, and fast computation [39]. If we consider a time series $\left\{x_{1}, x_{2}, \ldots, x_{N}\right\}$ and assume $X_{i}=\left(X_{i}, X_{i+J}, \ldots, X_{i+(m-t) J}\right)$ be the system state at discrete time $i$, where $J$ is the lag or reconstruction delay and $m$ is the embedding dimension, then, the reconstructed trajectory $\mathbf{X}$ is $\mathbf{X}=\left(\mathrm{X}_{1} \mathrm{X}_{2} \ldots\right.$ $\left.\mathrm{X}_{M}\right)^{\mathrm{T}}$. Subsequently, under this approach we depend on localizing the nearest neighbor of each point of the reconstructed trajectory $\mathbf{X}$. For instance, the nearest neighbor $\mathbf{X}_{j}$ to $\mathbf{X}_{i}$ is found by finding the smallest distance $d_{\mathrm{j}}(0)$ given by [39]

$$
d_{j}(0)=\min _{\mathbf{x}_{j}}\left\|\mathbf{X}_{j}-\mathbf{X}_{i}\right\|
$$


Then, the LE denoted $\lambda$ of the series is given as

$d(t)=C e^{\lambda_{1} t}$

where $d(t)$ is the average divergence at time $t$ of the underlying time series and $C$ is a constant that normalizes the initial separation. Assuming that the $j$ th pair of nearest neighbors diverges approximately at a rate given by the LE we get

$$
d_{j}(i) \approx C_{j} e^{\lambda_{1}(i \Delta t)}
$$

where $\Delta t$ is the sampling period and $d_{j}(i)$ the distance between the $j$ th pair of nearest neighbors after $i$ discrete-time steps. By taking the logarithm on both sides of Eq.12, the following is obtained

$\log d_{j}(t) \approx \log C_{j}+\lambda_{1}(i \Delta t)$

Indeed, Eq.13 corresponds to a set of approximately parallel lines (for $j=1,2, \ldots, M$ ), each with a slope approximately proportional to $\lambda_{1}$. Then, the LE is estimated by using least-squares fit to the average line expressed as

$$
y(i)=\frac{1}{\Delta t}\left\langle\log d_{j}(i)\right\rangle
$$

where $\langle\cdot\rangle$ denotes the average over all values of $j$. Following the recommendation of Rosenstein et al [39], we assume that the reconstruction delay $J$ corresponds to the lag before the first decline of the autocorrelation function, and the embedding dimension $m$ is determined based on the smallest value that allows convergence. This way fast computation is also enhanced.

\section{EXTENSIVE COMPARATIVE SURVEY}

We explore the nonlinear dynamical properties of eight (8) Islamic and Green crypto-currencies including X8X (Islamic), OneGram (Islamic), BitcoinGreen, GreenX, GreenMed, EverGreenCoin, GreenCoin, and Greenenergy Token. Each time period we employ varies with the availability of the data. For instance, it spans from 29 January 2018 to 23 May 2019 for the X8X, 07 December 2018 to 13 May 2019 for OneGram, 15 April 2018 to 23 May 2019 for BitcoinGreen, 01 September 2018 to 23 May 2019 for GreenX, 04 February 2018 to 23 May 2019 for GreenMed, 18 January 2016 to 23 May 2019 for EverGreenCoin, 02 February 2015 to 23 May 2019 for GreenCoin, and 27 November 2018 to 29 April 2019 for Greenenergy Token. For comparative 
purposes, another empirical investigation is conducted as well. The period includes 29 January 2018 to 23 May 2019 and the database is composed of seventeen (17) non-Islamic and nongreen crypto-currencies namely, Augur, Bitcoin, Cardano, EOS, Ethereum, Litecoin, Metaverse, Monero, NEO, OmiseGo, OX, QTUM, Ripple, Stellar, Tether, Tronix, and Zcash. Overall, the long memory is examined in prices, returns, volumes and volatilities of twenty-five (25) cryptocurrencies. We graphically depict the time series in Fig.1 (0X0, Islamic crypto-currency), Fig.2 (GreenEnergy Token, green crypto-currency) and Fig.3 (Bitcoin, non-Islamic and non-green crypto-currency).
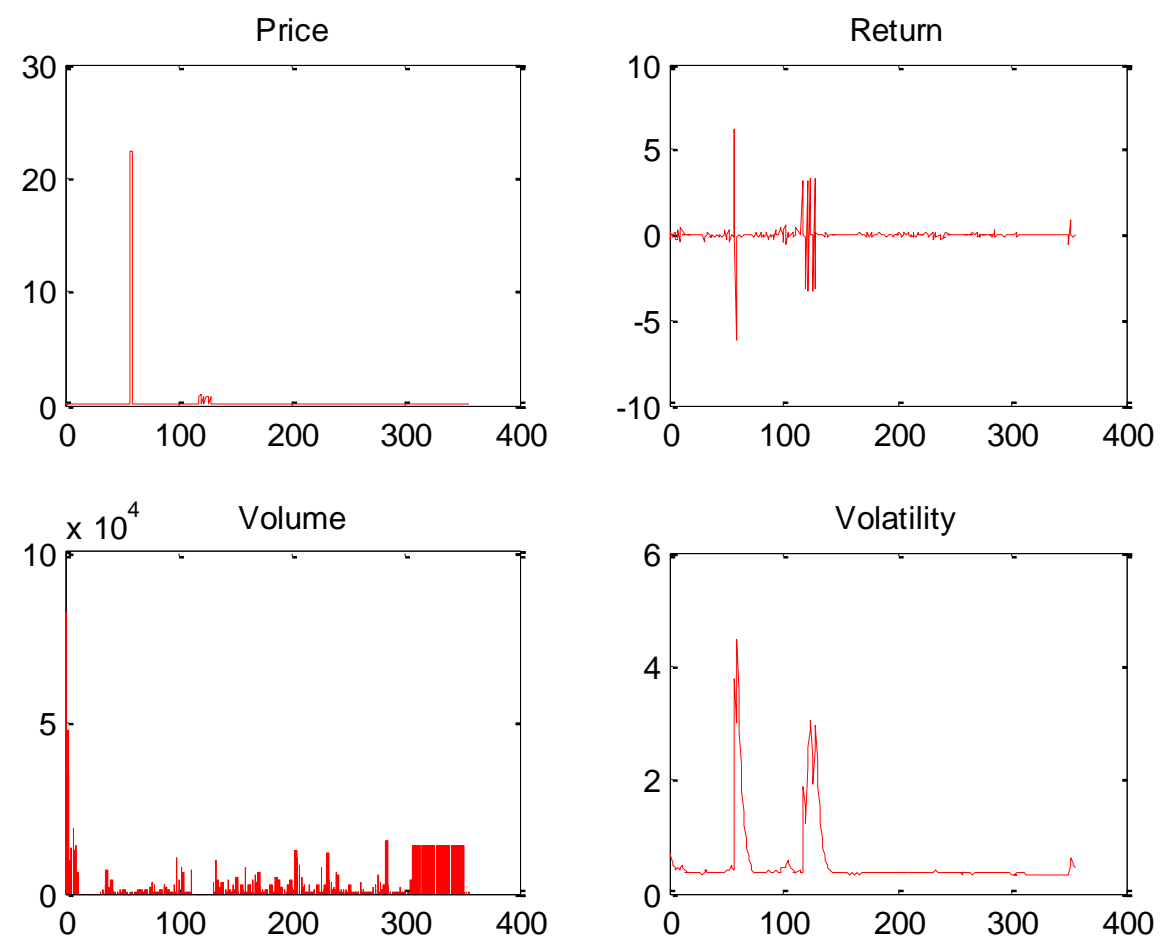

Fig.1. Plots of 0X0 (Islamic crypto-currency) series 

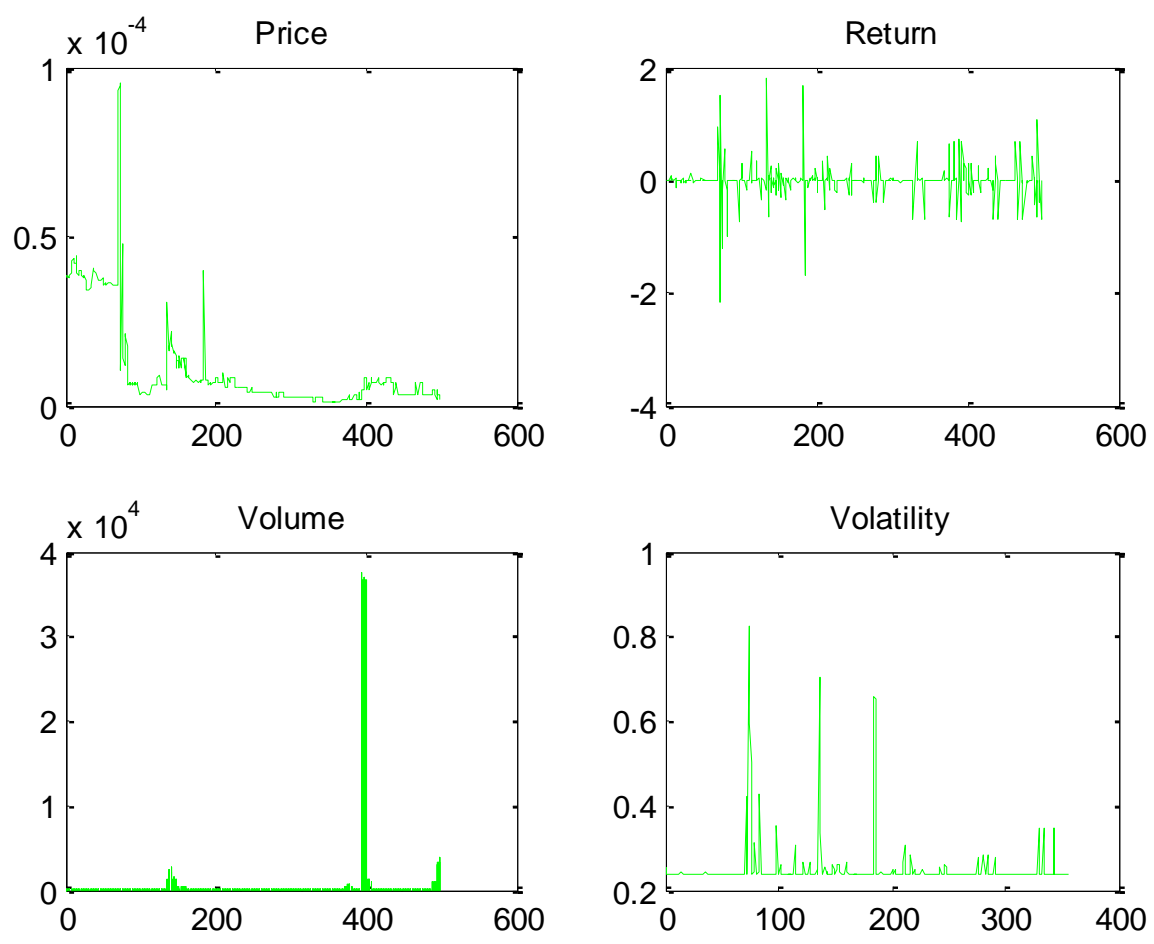

Fig.2. Plots of GreenEnergy Token (Green crypto-currency) series
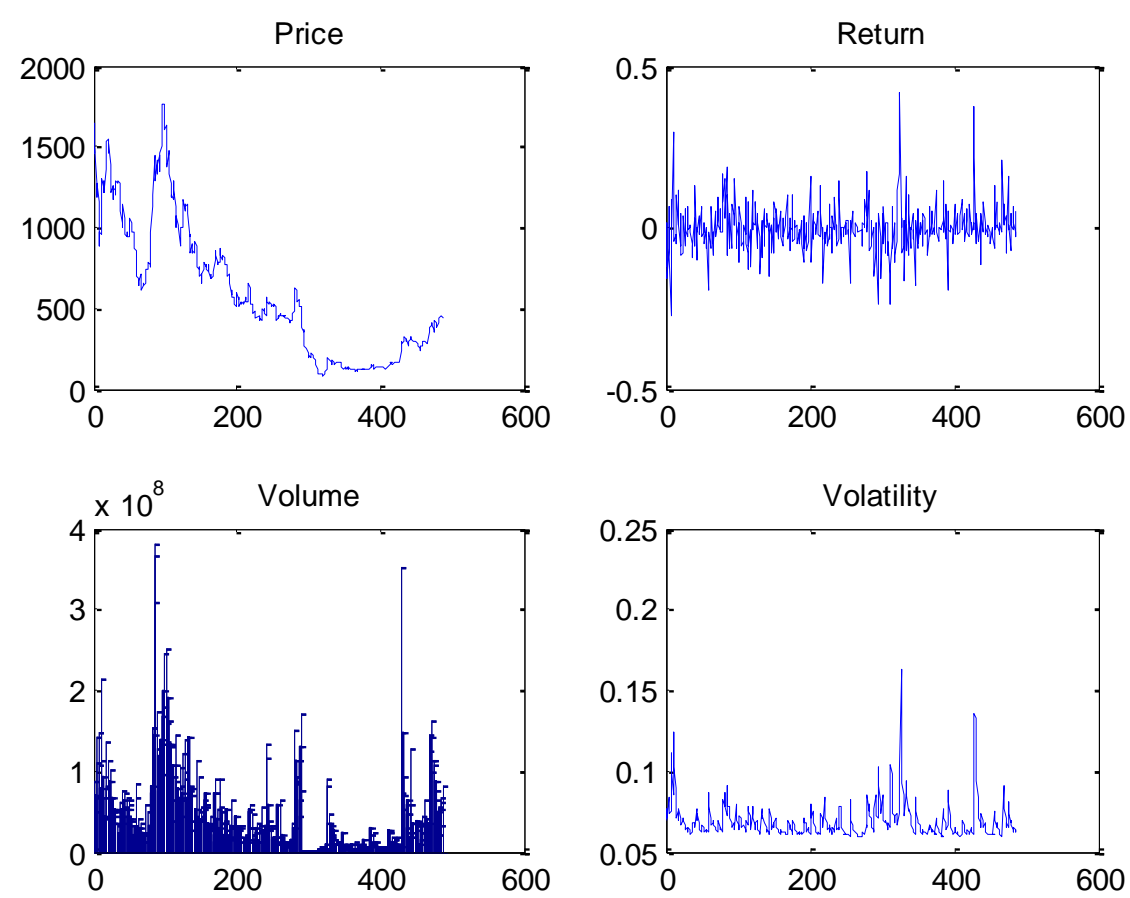

Fig.3. Plots of Bitcoin (common crypto-currency) series 
In Fig. 4 we show the distribution of estimated long memory parameters captured by the HE (for prices returns and volume) while FIGARCH-based parameters $d$ for the volatility series by groups (Islamic and green versus others), all of which in boxplots. We can observe that the Hurst exponent (HE) related to prices and volume time series is largely above 0.5 for the Islamic and Green crypto-currencies as well as for non-Islamic and non-green ones. Thus, long memory is highly persistent in these two different categories of crypto-currencies. In addition, as illustrated the median of the HE is below 0.5 for Islamic and green crypto-currencies and above 0.5 for the non-Islamic and non-green ones. The returns in Islamic and green crypto-currencies exhibit anti-persistent dynamics, whilst for non-Islamic and non-green crypto-currencies persistent dynamics emerge in our results. In case of the volatility, the median of the distribution of the estimated parameter $d$ in Islamic and green crypto-currencies is close to unity, i.e., the conditional second-moment demonstrates fully-integrated persistence. On the contrary, non-Islamic and non-green volatility medians are close to zero, which in turn signifies decay under a geometric rate.

Moreover, as seen in Fig.4, the distributions of estimated long memory parameters are different between Islamic and green ones as well as among common crypto-currencies. The differences are clear especially considering prices, return, and volatility. In order to formally investigate the significance of the output, a Student's $t$-test (one sided and two-sided) and an $F$ test are applied to the populations of the estimated HE and $d$ parameters. All statistical tests are performed at $5 \%$ statistical significance level. The results are presented in Table 1 and Table 2 respectively. According to the Student's $t$-test shown in Table 1, when the computed $p$-value is less than $5 \%$, the null hypothesis of equality of means (two-sided) in the HE populations is rejected for the return time series while the null for the $d$ parameter is rejected only for the volatility. Therefore, the long memory presence in the returns and volatility series is statistically different in-between the groups composed of Islamic and green and non-Islamic and non-green cryptos. In addition, according to the calculated $p$-values associated with the one-sided Student's $t$-test for the HEs in prices, returns and volume in case of Islamic and green cryptocurrencies is larger than that from non-Islamic and non-green ones. 


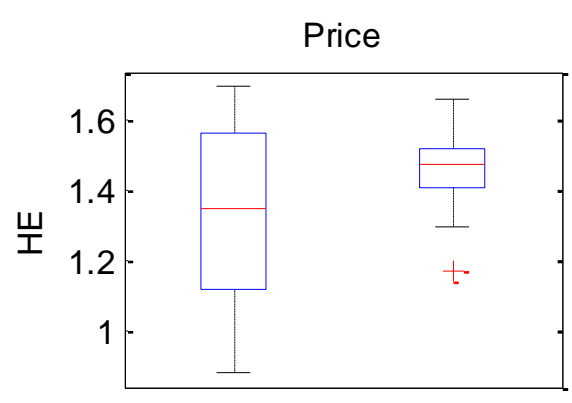

Islamic \& Green Others

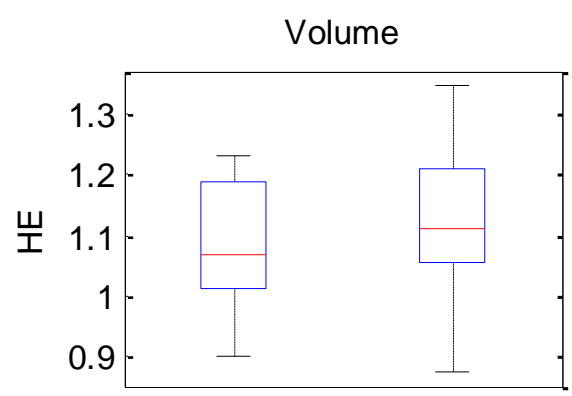

Islamic \& Green Others

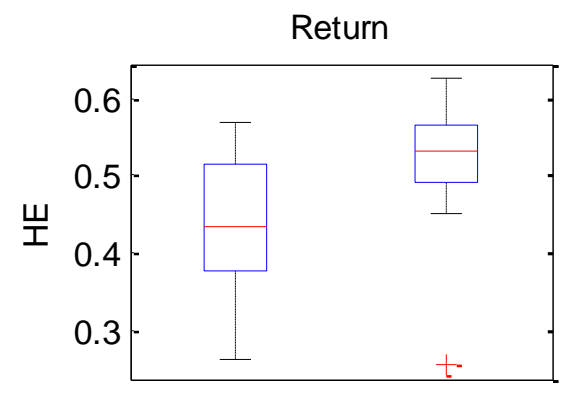

Islamic \& Green Others

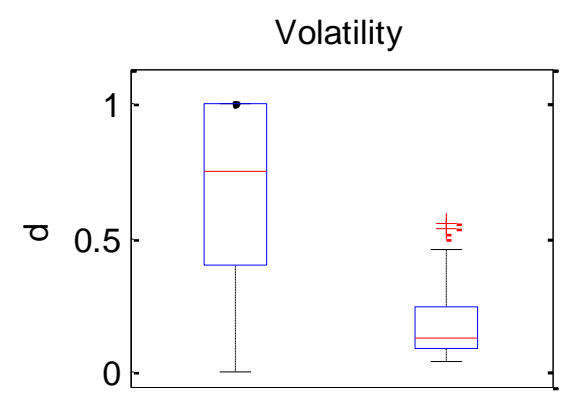

Islamic \& Green Others

Fig.4. Boxplots of estimated long memory parameters in crypto-currencies. The multi-step nonlinear filter estimates the HE via the DFA and the $d$ from a FIGARCH-based process. The horizontal red line inside the box indicates the median.

Next, as inferred from the $p$-values from the two-sided $F$-test shown in Table 2 regarding the null of equality of variances, the variability of the estimated HE from price series for the group composed of Islamic and green crypto-currencies is different from the one estimated for the common crypto-currencies. Similarly, the variability of $d$ estimated by the volatility series of the group composed of Islamic and green crypto-currencies is different from the variance of the $d$ in prices detected in the group composed of common crypto-currencies. Overall, the calculated $p$ values associated with the one-sided $F$-test show strong evidence that the variability in $\mathrm{HE}(d)$ in prices (volatility) of Islamic and green crypto-currencies is lower vis-à-vis the one from common crypto-currencies.

Table.1: Student $t$-test applied to DFA-based HE

\begin{tabular}{lcccc}
\hline \multicolumn{1}{c}{ Series } & Hypothesis & $\boldsymbol{p}$-value & Hypothesis & $\boldsymbol{p}$-value \\
\hline Price & $\mathrm{HE}_{\text {Islamic\&Green }}=\mathrm{HE}_{\text {Others }}$ & 0.1143 & $\mathrm{HE}_{\text {Islamic\&Green }}>\mathrm{HE}_{\text {Others }}$ & 0.9428 \\
Return & $\mathrm{HE}_{\text {Islamic\&Green }}=\mathrm{HE}_{\text {Others }}$ & 0.0405 & $\mathrm{HE}_{\text {Islamic\&Green }}>\mathrm{HE}_{\text {others }}$ & 0.9797 \\
Volume & $\mathrm{HE}_{\text {Islamic\&Green }}=\mathrm{HE} \mathrm{E}_{\text {Others }}$ & 0.3787 & $\mathrm{HE}_{\text {Islamic\&Green }}>\mathrm{HE}_{\text {Others }}$ & 0.8106 \\
Volatility & $d_{\text {Islamic\&Green }}=d_{\text {Others }}$ & 0.0000 & $d_{\text {Islamic\&Green }}>d_{\text {Others }}$ & 0.0000 \\
\hline
\end{tabular}


Table.2: F-test applied to DFA-based HE

\begin{tabular}{|c|c|c|c|c|}
\hline Series & Hypothesis & $p$-value & Hypothesis & $p$-value \\
\hline Price & $\mathrm{HE}_{\text {Islamic\&Green }}=\mathrm{HE}_{\text {Others }}$ & 0.0040 & $\mathrm{HE}_{\text {Islamic\&Green }}>\mathrm{HE}_{\text {others }}$ & 0.0020 \\
\hline Return & $\mathrm{HE}_{\text {Islamic\&Green }}=\mathrm{HE}_{\text {Others }}$ & 0.5587 & $\mathrm{HE}_{\text {Islamic\&Green }}>\mathrm{HE}_{\text {Others }}$ & 0.2794 \\
\hline Volume & $\mathrm{HE}_{\text {Islamic\&Green }}=\mathrm{HE}_{\text {Others }}$ & 0.8047 & $\mathrm{HE}_{\text {Islamic\&Green }}>\mathrm{HE}_{\text {others }}$ & 0.5977 \\
\hline Volatility & $d_{\text {Islamic\&Green }}=d_{\text {Others }}$ & 0.0076 & $d_{\text {Islamic\&Green }}>d_{\text {Others }}$ & 0.0038 \\
\hline
\end{tabular}

The boxplots of the averages of GHE estimates across all scales $(-10 \leq q \leq 10)$ are presented in Fig.5. The plots suggest that the distributions of GHE estimates differ among the groups of Islamic, green and common crypto-currencies. This point is also asserted when looking the results from the Student and F- tests presented respectively in Tables 3 and 4 . Whilst according to the calculated $p$-values, the GHE estimates of all series are different between the two groups, yet this will not apply to price series. In addition, the GHE estimates of all series from the first group (Islamic \& green) are higher compared to those of the second group, apart from for prices and returns. Moreover, based on the computed $p$-values illustrated in Table 4 , the variance of the GHE estimates of all series is across groups, except for the return series. Additionally, the variance of the GHEs in the first group is higher, only aside from returns. Consequently, while both groups exhibit multi-fractality, Islamic and green crypto-currencies demonstrate a significantly higher degree of inherent fractality than common cryptos.

Table.3: Student $t$-test applied to averages of GHE estimates

\begin{tabular}{|c|c|c|c|c|}
\hline Series & Hypothesis & $p$-value & Hypothesis & $p$-value \\
\hline Price & $\mathrm{LE}_{\text {Islamic\&Green }}=\mathrm{LE}_{\text {Otherrs }}$ & 0.1290 & $\mathrm{LE}_{\text {Islamic\&Green }}>\mathrm{LE}_{\text {Others }}$ & 0.0645 \\
\hline Return & $\mathrm{LE}_{\text {Islamic\&Green }}=\mathrm{LE}_{\text {Others }}$ & 0.0000 & $\mathrm{LE}_{\text {Islamic\&Green }}>\mathrm{LE}_{\text {Others }}$ & 0.9999 \\
\hline Volume & $\mathrm{LE}_{\text {Islamic\&Green }}=\mathrm{LE}_{\text {Others }}$ & 0.0000 & $\mathrm{LE}_{\text {Islamic\&Green }}>\mathrm{LE}_{\text {Others }}$ & 0.0000 \\
\hline Volatility & $\mathrm{LE}_{\text {Islamic\&Green }}=\mathrm{LE}_{\text {Others }}$ & 0.0122 & $\mathrm{LE}_{\text {Islamic\&Green }}=\mathrm{LE}_{\text {Others }}$ & 0.0061 \\
\hline
\end{tabular}

Table.4: $F$-test applied to averages of GHE estimates

\begin{tabular}{|c|c|c|c|c|}
\hline Series & Hypothesis & $p$-value & Hypothesis & $p$-value \\
\hline Price & $\mathrm{LE}_{\text {Islamic\&Green }}=\mathrm{LE}_{\text {Otherrs }}$ & 0.0000 & $\mathrm{LE}_{\text {Islamic\&Green }}>\mathrm{LE}_{\text {Others }}$ & 0.0000 \\
\hline Return & $\mathrm{LE}_{\text {Islamic\&Green }}=\mathrm{LE}_{\text {Others }}$ & 0.1482 & $\mathrm{LE}_{\text {Islamic\&Green }}>\mathrm{LE}_{\text {Others }}$ & 0.0741 \\
\hline Volume & $\mathrm{LE}_{\text {Islamic\&Green }}=\mathrm{LE}_{\text {Others }}$ & 0.0000 & $\mathrm{LE}_{\text {Islamic\&Green }}>\mathrm{LE}_{\text {Others }}$ & 0.0000 \\
\hline Volatility & $\mathrm{LE}_{\text {Islamic\&Green }}=\mathrm{LE}_{\text {Others }}$ & 0.0000 & $\mathrm{LE}_{\text {Islamic\&Green }}=\mathrm{LE}_{\text {Others }}$ & 0.0000 \\
\hline
\end{tabular}




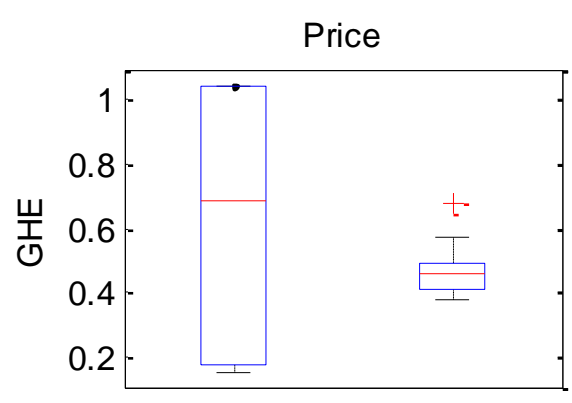

Islamic \& Green Others

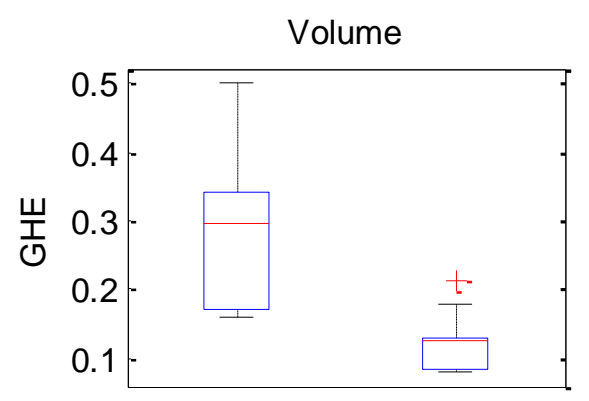

Islamic \& Green Others

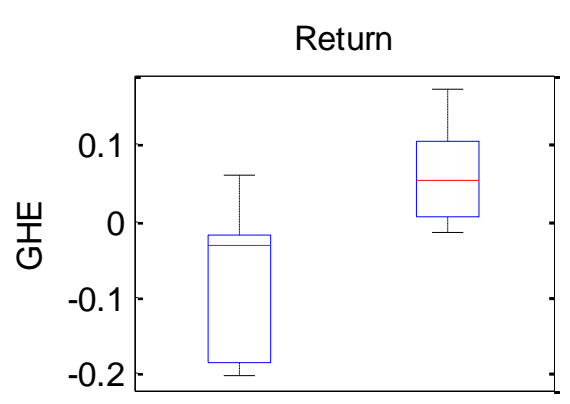

Islamic \& Green Others

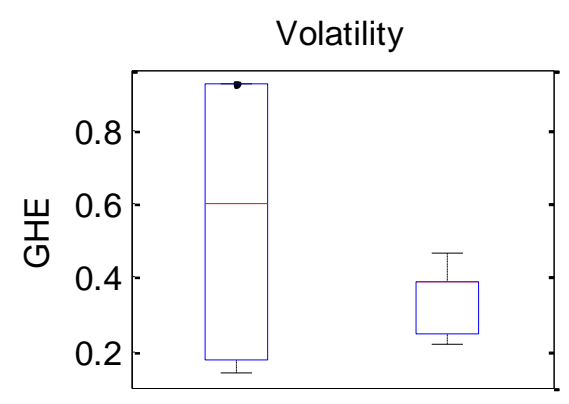

Islamic \& Green Others

Fig.5. Boxplots of estimated Generalized Hurst exponent (GHE) in crypto-currencies. The multi-step nonlinear filter estimates the LE based on a hybrid synthetic DFA approach. The horizontal red line inside the box indicates the median.

Fig.6 displays the boxplots of Lyapunov exponents (LE). At first sight, the LE estimates of Islamic, green versus conventional crypto-currencies, derive from different distributions. Specifically, the $p$-values obtained from Student $t$-test (Table 5) indicate that the mean of LE in prices is different across Islamic, green and conventional crypto-currencies groups. The same applies to volatility series. Furthermore, the $p$-values from the $F$-test vis-à-vis the equality of variances for these chaotic estimates (Table 6) show evidence that the variance of the LEs for the first group is significantly different from that of conventional cryptos. If we exclude the return series, the variance of the LEs in the first group is fundamentally higher.

Table.5: Student $t$-test applied to LE estimates

\begin{tabular}{|c|c|c|c|c|}
\hline Series & Hypothesis & $p$-value & Hypothesis & $p$-value \\
\hline Price & $\mathrm{LE}_{\text {Islamic\&Green }}=\mathrm{LE}_{\text {Otherrs }}$ & 0.0000 & $\mathrm{LE}_{\text {Islamic\&Green }}>\mathrm{LE}_{\text {Others }}$ & 1 \\
\hline Return & $\mathrm{LE}_{\text {Islamic\&Green }}=\mathrm{LE}_{\text {Others }}$ & 0.7284 & $\mathrm{LE}_{\text {Islamic\&Green }}>\mathrm{LE}_{\text {Others }}$ & 0.3642 \\
\hline Volume & $\mathrm{LE}_{\text {Islamic\&Green }}=\mathrm{LE}_{\text {Others }}$ & 0.0903 & $\mathrm{LE}_{\text {Islamic\&Green }}>\mathrm{LE}_{\text {Others }}$ & 0.0452 \\
\hline Volatility & $L_{\text {Islamic\&Green }}=L_{E_{\text {Others }}}$ & 0.0495 & $L_{\text {Islamic\&Green }}=L_{\text {Others }}$ & 0.0248 \\
\hline
\end{tabular}


Table.6: $F$-test applied to LE estimates

\begin{tabular}{llccc}
\hline \multicolumn{1}{c}{ Series } & \multicolumn{1}{c}{ Hypothesis } & $\boldsymbol{p}$-value & Hypothesis & $\boldsymbol{p}$-value \\
\hline Price & $\mathrm{LE}_{\text {Islamic\&Green }}=\mathrm{LE}_{\text {Otherrs }}$ & 0.0050 & $\mathrm{LE}_{\text {Islamic\&Green }}>\mathrm{LE}_{\text {others }}$ & 0.0022 \\
Return & $\mathrm{LE}_{\text {Islamic\&Green }}=\mathrm{LE}_{\text {Others }}$ & 0.0024 & $\mathrm{LE}_{\text {Islamic\&Green }}>\mathrm{LE}_{\text {others }}$ & 0.9988 \\
Volume & $\mathrm{LE}_{\text {Islamic\&Green }}=\mathrm{LE}_{\text {Others }}$ & 0.0042 & $\mathrm{LE}_{\text {Islamic\&Green }}>$ LE & 0.0021 \\
Volathers & 0.0018 \\
\hline
\end{tabular}

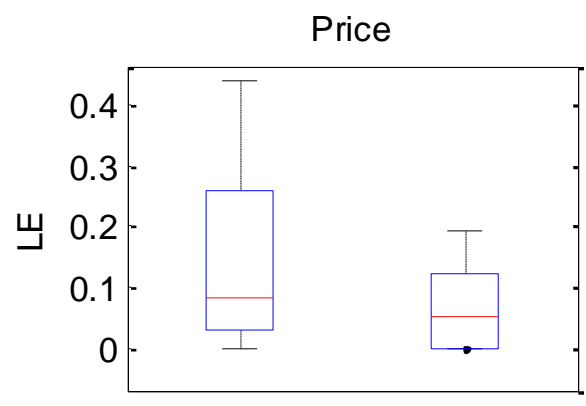

Islamic \& Green Others

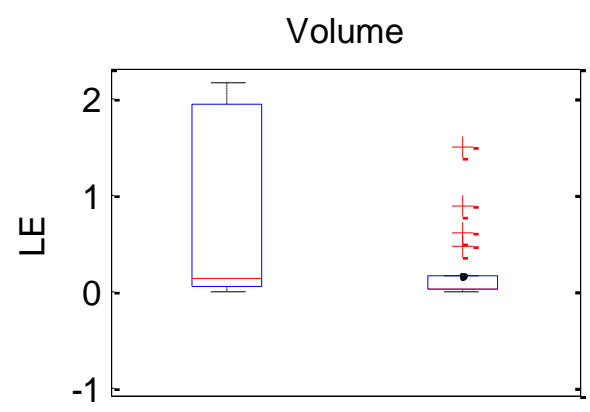

Islamic \& Green Others

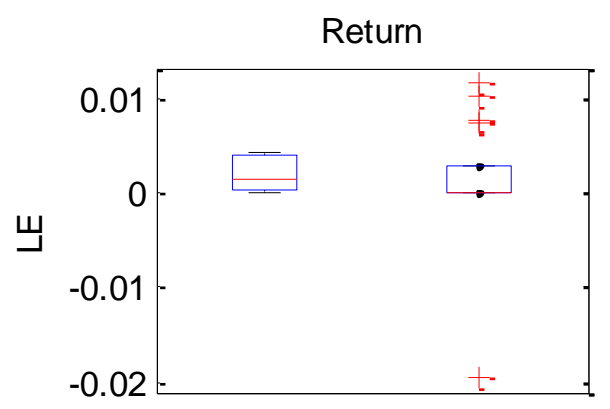

Islamic \& Green Others

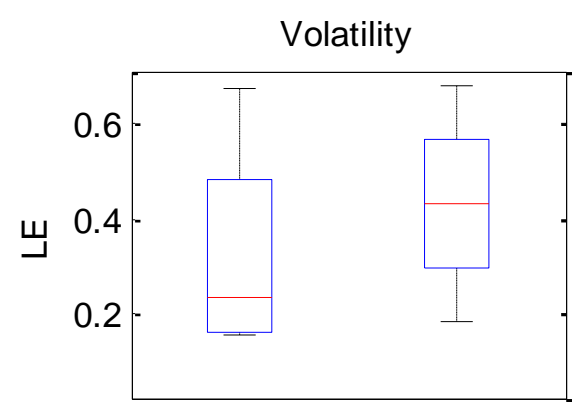

Islamic \& Green Others

Fig.6. Boxplots of estimated Lyapunov exponent (LE) in crypto-currencies. The multi-step nonlinear filter estimates the LE based on the Rosenstein approach. The horizontal red line inside the box indicates the median.

To sum-up, the exhaustive statistical investigation reveals that returns in Islamic and green crypto-currencies exhibit anti-persistent dynamics. Prices, returns and volume series from Islamic and green crypto-currencies show a higher level of persistence in comparison to common crypto-currencies. Furthermore, the variability of the long memory behaviour in price and volatility series is diverse between the two groups we examined. The level of variability in persistence for price and volume series in case of Islamic and green crypto-currencies is lower. Our findings expose heterogeneity in long memory patterns of Islamic and green vis-à-vis the common crypto-currencies. While both groups inherit multi-fractal dynamics, for Islamic and 
green crypto-currencies is more profound. The outcome of survey statistical testing performed on all chaotic parameters is conclusive of the observed heterogeneity among the various groups. Hence, the distributions of the parameters are clearly modular and significantly diverse. In that, forecastability may be achievable at very short horizons for all cryptos due to chaoticity, but the nonlinear predictability of the Islamic and Green group is particularly profound with distinct chaotic long-memory traits, hence far more interesting for investors.

\section{ConClusions}

We comparatively investigate long memory, multi-fractality and chaoticity in Islamic and Green cryptos versus common ones. We analyse the patters of prices, returns, volume and volatilities following a hybrid decomposition approach incorporating detrended fluctuation analysis, generalized Hurst and Lyapunov exponents and second-moment FIGARCH processing. The resulting estimates are compared and dissimilarities are revealed. The proposed multi-step dynamic filtering may further contribute to better understanding of the underlying nonlinear structure of Islamic and Green cryptocurrency markets, which very recently have gained a growing interest by traders, speculators and financial institutions.

\section{REFERENCES}

[1] A. Phillip, J.S.K. Chan, S. Peiris, A new look at Cryptocurrencies, Economics Letters 163 (2018) 6-9.

[2] G.M. Caporale, L. Gil-Alanac, A. Plastun, Persistence in the cryptocurrency market, Research in International Business and Finance 46 (2018) 141-148.

[3] L. Catania, S. Grassi, F. Ravazzolo, Predicting the volatility of cryptocurrency time-series, In: Corazza M., Durbán M., Grané A., Perna C., Sibillo M. (eds) Mathematical and Statistical Methods for Actuarial Sciences and Finance. Springer, Cham, 203-207.

[4] J. Alvarez-Ramirez, E. Rodriguez, C. Ibarra-Valdez, Long-range correlations and asymmetry in the Bitcoin market, Physica A 492 (2018) 948-955.

[5] W. Zhang, P. Wang, X. Li, D. Shen, Some stylized facts of the cryptocurrency market, Applied Economics 50 (2018) 5950-5965.

[6] C. Conrad, A. Custovic, E. Ghysels, Long- and short-term cryptocurrency volatility components: A GARCH-MIDAS Analysis, Journal of Risk and Financial Management 11 (2018) 1-12.

[7] J. Chu, S. Chan, S. Nadarajah, J. Osterrieder, GARCH Modelling of Cryptocurrencies, Journal of Risk and Financial Management 10 (2017). https://doi.org/10.3390/jrfm10040017 
[8] P. Katsiampa, Volatility estimation for Bitcoin: A comparison of GARCH models, Economics Letters 158 (2017) 3-6.

[9]A. Haubo Dyhrberg, Bitcoin, gold and the dollar - A GARCH volatility analysis, Finance Research Letters 16 (2016) 85-92.

[10] A. Haubo Dyhrberg, Hedging capabilities of bitcoin. Is it the virtual gold? Finance Research Letters 16 (2016) 139-144.

[11] P. Ciaian, M. Rajcaniova, d'Artis Kancs, Virtual relationships: Short- and long-run evidence from BitCoin and altcoin markets, Journal of International Financial Markets, Institutions and Money 52 (2018) 173-195.

[12] P. Ciaian, M. Rajcaniova, d'Artis Kancs, The economics of BitCoin price formation, Applied Economics 48 (2016) 1799-1815.

[13] M. Polasik, A.I. Piotrowska, T.P. Wisniewski, R. Kotkowski, G. Lightfoot, Price Fluctuations and the Use of Bitcoin: An Empirical Inquiry, International Journal of Electronic Commerce 20 (2015) 9-49.

[14] P. Bação, A. Portugal Duarte, H. Sebastião, S. Redzepagic, Information transmission between cryptocurrencies: Does Bitcoin rule the cryptocurrency world? Scientific Annals of Economics and Business 65 (2018) 97-117.

[15] A.F. Bariviera, L. Zunino, O.A. Rosso, An analysis of high-frequency cryptocurrencies prices dynamics using permutation-information-theory quantifiers, Chaos 28, 075511 (2018) https://doi.org/10.1063/1.5027153

[16] A. Phillip, J. Chan, S. Peiris, On long memory effects in the volatility measure of Cryptocurrencies, Finance Research Letters 28 (2019) 95-100.

[17] S. Lahmiri, S. Bekiros, A. Salvi, Long-range memory, distributional variation and randomness of bitcoin volatility, Chaos, Solitons \& Fractals 107 (2018) 43-48.

[18] S. Stavroyiannis, V. Babalos, S. Bekiros, S. Lahmiri, G.S. Uddin, The high frequency multifractal properties of Bitcoin, Physica A 520 (2019) 62-71.

[19] S. Lahmiri, S. Bekiros, Chaos, randomness and multi-fractality in Bitcoin market, Chaos, solitons \& fractals 106 (2018) 28-34.

[20] L. Alessandretti, A. ElBahrawy, L. Maria Aiello, A. Baronchelli, Anticipating Cryptocurrency Prices Using Machine Learning, Complexity (2018) Article ID 8983590, 16 pages, https://doi.org/10.1155/2018/8983590

[21] S. Lahmiri, S. Bekiros, Cryptocurrency forecasting with deep learning chaotic neural networks, Chaos, Solitons \& Fractals 118 (2019) 35-40.

[22] S. Ali, S.J.H. Shahzad, N. Raza, K.H. Al-Yahyaee, Stock market efficiency: A comparative analysis of Islamic and conventional stock markets, Physica A 503 (2018) 139-153.

[23] C. Aloui, R. Jammazi, H. Ben Hamida, Multivariate Co-movement Between Islamic Stock and Bond Markets Among the GCC: A Wavelet-Based View, Computational Economics August 52 (2018) 603-626. 
[24] S. Lahmiri, S. Bekiros, Time-varying self-similarity in alternative investments, Chaos, Solitons \& Fractals 111 (2018) 1-5.

[25] T. Mezghani, M. Boujelbène, The contagion effect between the oil market, and the Islamic and conventional stock markets of the GCC country: Behavioral explanation, International Journal of Islamic and Middle Eastern Finance and Management 11 (2018) 157-181.

[26] F. Saâdaoui, Testing for multifractality of Islamic stock markets, Physica A 496 (2018) 263-273.

[27] S. Lahmiri, S. Bekiros, S. Stavroyiannis, V. Babalos, Modelling volatility persistence under stochasticity assumptions: evidence from common and alternative investments, Chaos, Solitons \& Fractals 114 (2018) 158-163.

[28] F. Climent, P. Soriano, Green and Good? The Investment Performance of US Environmental Mutual Funds, Journal of Business Ethics 103 (2011) 275-287.

[29] C.E. Chang, W.A. Nelson, H.D. Witte, Do green mutual funds perform well? Management Research Review 35 (2012) 693-708.

[30] J. Robinson, A. Glean, W. Moore, How does news impact on the stock prices of green firms in emerging markets? Research in International Business and Finance 45 (2018) 446-453.

[31] J.C. Reboredo, Green bond and financial markets: Co-movement, diversification and price spillover effects, Energy Economics 74 (2018) 38-50.

[32] B. Scholtens, The sustainability of green funds, Natural resources forum 35 (2011) 223-232.

[33] C.-K. Peng, S.V. Buldyrev, S. Havlin, M. Simons, H.E. Stanley, A.L. Goldberger, Mosaic organization of DNA nucleotides, Phys. Rev. E 49 (1994) 1685-1689.

[34] R.T. Baillie, T. Bollerslev, H.O. Mikkelsen, Fractionally integrated generalized autoregressive conditional heteroskedasticity, Journal of Econometrics, 74 (1996), 3-30.

[35] M. Costa, A.L. Goldberger, C.K. Peng, Multiscale entropy analysis of complex physiologic time series, Physical Review Letters 89 (2002) 068102.

[36] J.-M. Bardet, I. Kammoun, Asymptotic properties of the detrended fluctuation analysis of long-rangedependent processes, IEEE Transactions on Information Theory 54 (2008) 2041-2052.

[37] M.S. Taqqu, V. Teverovsky, W. Willinger, Estimators for long-range dependence: an empirical study, Fractals 3 (1995) 785-798.

[38] R. Liu, T. Di Matteo, Thomas Lux, True and apparent scaling: The proximity of the Markov-switching multifractal model to long-range dependence, Physica A 383 (2007) 35-42.

[39] M.T. Rosenstein, J.J. Collins, C.J. De Luca, A practical method for calculating largest Lyapunov exponents from small data sets, Physica D 65 (1993) 117-134. 